\title{
Design of Intelligent Traffic Control System Based on Fuzzy Algorithm
}

\author{
Li Yong \\ Road \& Bridge North China engineering CO., LTD. China Beijing 101100
}

Keywords: fuzzy algorithm; SQLite database; intelligent traffic control

\begin{abstract}
An intelligent traffic control system based on fuzzy algorithm is designed. The functions of the system includes traffic signal control, fuzzy algorithm control, and control parameter settings, and also introduced the SQLite database as a system repository.
\end{abstract}

\section{Introduction}

With the development of people's living standards and car production efficiency, more and more people could buy cars. However, the speed of construction of traffic roads is much smaller than the speed of people buying cars, which will inevitably lead to urban traffic problems. How to solve the relationship between people, cars and roads is an urgent problem to be resolved. With the continuous application of artificial intelligence technology, applying it to traffic management can solve some traffic congestion problems. Due to the randomness and uncertainty of the traffic flow at the intersection, numerous scholars at home and abroad have conducted a lot of researches. Literature $^{[1-2]}$ uses time series algorithm to control traffic control. The designed time series algorithm can control most traffic, but due to the randomness of traffic flow, the prediction effect of this algorithm cannot be improved. In [3], the fuzzy algorithm is applied to intelligent traffic control. The simulation results demonstrate that the method can reduce the average queue length of intersections, thus improving the utilization of intersections. The text utilizes fuzzy algorithm as the core algorithm of intelligent traffic control system.

\section{Intelligent Traffic Control System Design System Requirements Analysis}

The main functions included in the intelligent traffic control system are: (1) traffic signals control. The principal contents are signal period, signal ratio and signal phase. The signal period is composed of red, yellow, and green color time. The cycle, of the signal light directly affects the traffic flow. If the traffic flow is heavy, the cycle is long and the cycle is short. Parameter settings directly affect traffic flow are the core technology of the system. The green letter ratio refers to the ratio of the cycle time of green time, and this value setting also affects the traffic flow. The phase difference is the right time difference that occurs after the two related phases are simultaneously activated, and this value can also affect the traffic flow. (2) Traffic signals control method. This article uses fixed time control, vehicle inspection and control of parameters. (3) Using fuzzy algorithms for intelligent control.

\section{Intelligent traffic control system designs}

\subsection{Overall system designs}

Intelligent traffic control system includes functions such as parameter setting, network communication, induction control, central coordination, wireless transmission, traffic light control, fault handling, application management, and fuzzy control. Figure 1 shows the overall function of the system. 


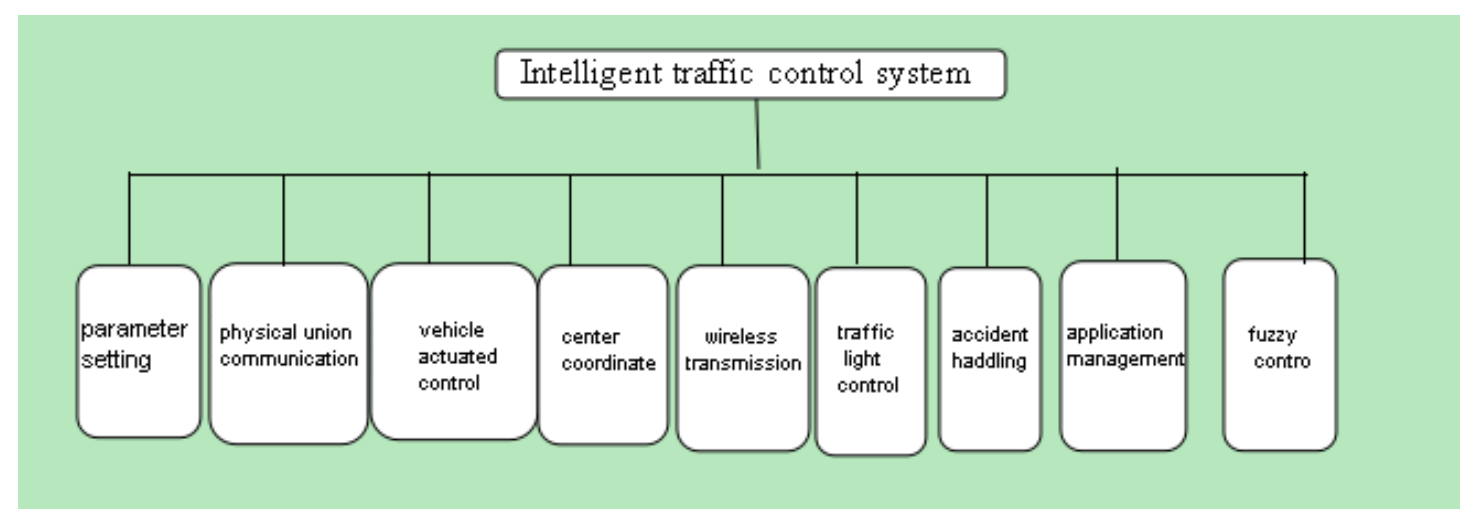

Figure 1. System overall function diagram

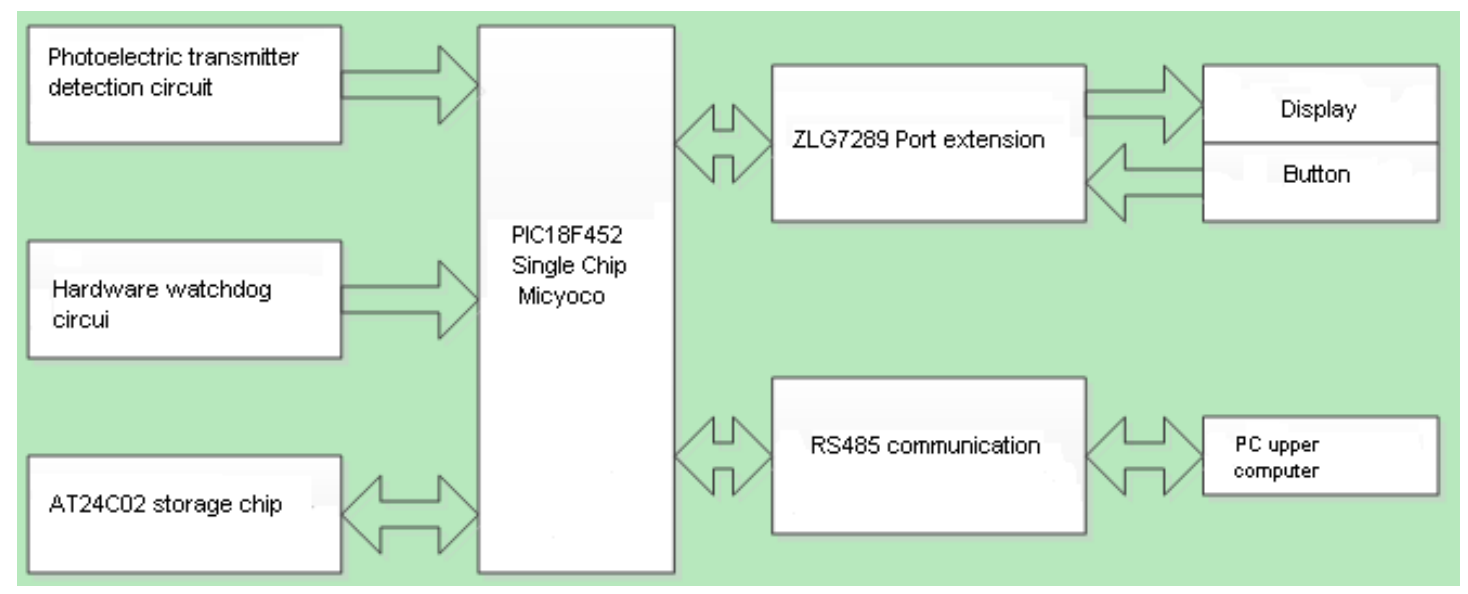

Figure 2. System hardware overall framework

The parameter setting module is primarily used for system parameter configuration such as signal period, signal ratio, and the like. Networked communication is used primarily for communication management. Inductive control is to control the corresponding device while traffic light control is for traffic control. Fuzzy control is the core algorithm of the system used to monitor traffic lights.

\subsection{System hardware designs}

The controller used throughout this paper is PIC18F452. The storage addressing of singlechip is $32 \mathrm{~KB}$ with the 16-bit wide instruction bus, which realizes the control by adding the fuzzy algorithm to the controller. Data transmission is performed using RS485 serial port. Figure 2 displays the overall frame diagram of the system hardware. An external memory chip AT24C02 has been added to increase the stability of the system, which can be used to store time ratios, control modes, and the like. ZLG7289 chip is to control the change of colours.

\subsection{System software designs}

\subsubsection{Fuzzy algorithm design}

\footnotetext{
Begin

Step1: Initialization. Find the maximum green time in the specified phase from phase j Gjmax

Step2: The minimum green time should meet $\mathrm{Gj} \geq 15, \mathrm{Gj}=\Delta \mathrm{G}$

Step3: Set the traffic flow in $\Delta \mathrm{G}$ as $\mathrm{Li}$

Step4: If $\mathrm{Li}<\mathrm{Gj}$, the green light needs to be converted to the next phase and jump to Step2 for operation

Step5: Green light $\Delta \mathrm{G}$ is set according to $\mathrm{Li}$. If there is a slight delay in green phase, $\mathrm{Li}$ increases. A fuzzy method should be established if green time should be increased. Let the total green time be $\Delta \mathrm{G}$, then $\mathrm{Gj}+\Delta \mathrm{G} \geq \mathrm{Gjmax}, \Delta \mathrm{G}=\mathrm{Gjmax}-\mathrm{Gj}$, otherwise $\mathrm{Gj}+\Delta \mathrm{G} \rightarrow \mathrm{Gj}$ jump to Step3 End
}

Inductive controllers are used to reduce vehicle delay errors when there is a sudden change in the 
heavy traffic flow. Experience has shown that the signal period for a single intersection should be greater than 15P (P is the number of phases), which avoids traffic accidents due to green time of less than 15s. Therefore, a single intersection fuzzy algorithm is designed [4-5]. The pseudo code of the algorithm is as follows:

\subsubsection{Software Framework of Control System}

In order to assist management and improve system stability, this paper chooses Linux system as the embedded development system. The system has high portability, open source code, and can save labor costs when acquiring the system. Figure 3 shows the intelligent traffic control software framework.

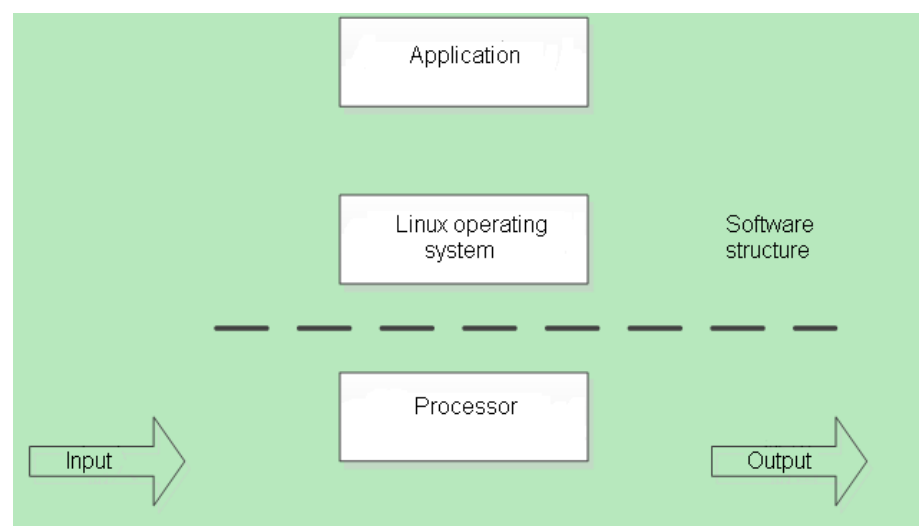

Figure 3 Intelligent traffic control software architecture diagram

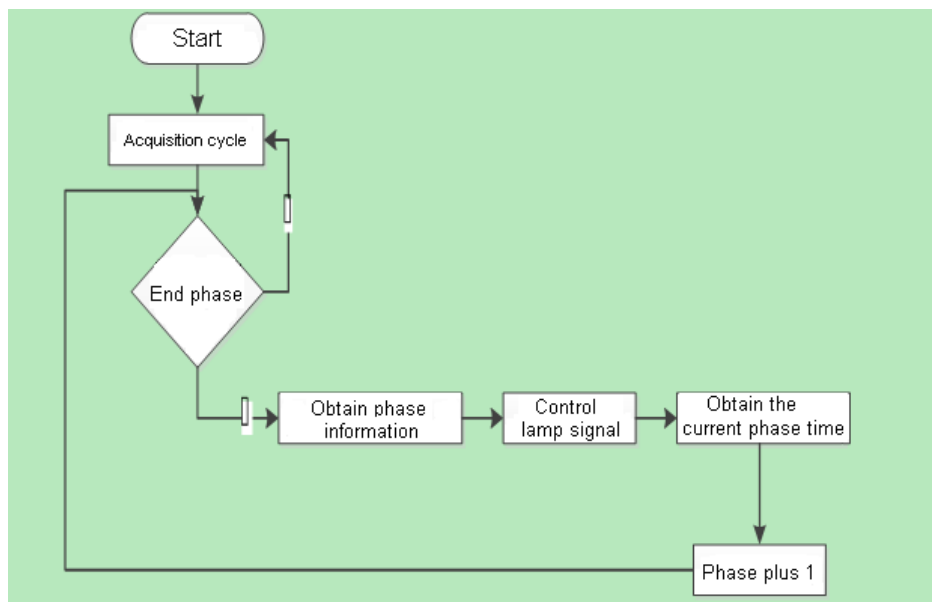

Figure 4 Single cycle timing control flow chart

As can be seen from Figure 3, intelligent traffic control software consists of two parts: the application layer and the operating system. The operating system hardware consists of a processor and input and output devices. The application layer contains numerous applications.

\subsubsection{Signal Control Program Design}

Signal control is at the heart function of the system. This control has to be combined with a fuzzy algorithm. The signal control flow is as follows: the first step is to acquire the cycle; then the phase judgment is performed, and the current phase is obtained according to the phase information; then the red, green and yellow light control is performed, and the process needs to set the corresponding time. Figure 4 shows the single cycle timing control flow chart.

\subsection{System database design}

The intelligent traffic control system is running all the time and will contain a lot of data. And this data has a focus on control, which needs to be stored for this purpose. SQLite is a lightweight database that is widely used in embedded systems, with the advantages of less resources and faster response time. This article chooses SQlite as a data storage set. The tables included in the intelligent 
traffic control are: a device information table, a control information table, and the like.

Table 1 shows the details of the device information table.

Table 1 device information table

\begin{tabular}{lllll}
\hline Name & Field representation & Type & Comment & Primary key \\
\hline device name & Equipment_name & Text & Text & Yes \\
device category & Equipment_type & text & Text & \\
Device parameters & Equipment_par & text & Text & \\
Device expiration date & Equipment_usedate & real & Floating & \\
& & & point & \\
Device purchase time & Equipment_buydate & date & Date & \\
\hline
\end{tabular}

The device information table is used to store device information. The included fields are: device name, device category, device parameters, device expiration date, device purchase time, and so on.

Control information table. The table contains fields such as control time, yellow light running time, red light running time, green light running time, control number, and control location. Table 2 shows the details of the control information table.

Table 2 Control Information Table

\begin{tabular}{lll}
\hline Field Representation & Type & Primary Key \\
\hline control_ID & Text & Yes \\
Control_time & Date & \\
Control_Y-time & Date \\
Control_R_time & Date \\
Control_G_time & Date \\
Control_where & Text \\
\hline
\end{tabular}

\section{References}

[1] Yong Zhang, Wei Guan. Complexity measurement of traffic flow time series[J]. Journal of Traffic and Transportation Engineering,2009(2) :89-92.

[2] Ji-dong YUAN, Zhi-hai WANG. An overview of Time Series Representation and Classification Algorithm[J]. Computer Science,2015,42(3) :1-7.

[3] Zhaoxin Gong. Research on Intelligent Traffic Control Algorithm Based on Fuzzy Logic[J]. Science Technology and Engineering, 2009, 9(18): 5603-5606.

[4] Xin Hui. Intelligent Traffic Control built on Neuron and Fuzzy Algorithm[J]. Journal of Highway and Transportation Research and Development, 2016(7): 257-259.

[5] Wei ZHANG, Ri-dong XIAO, Jing DENG. Dynamic Fuzzy Neural Network Based on Genetic Algorithm for Urban Expressway Entrance Ramp Control[J]. Journal of Highway and Transportation, 2017,34(2) :129-134. 